\title{
Transport through hybrid superconducting/ferromagnetic double-path junction
}

\author{
T.J.S. Facio ${ }^{a}$, P.A. Orellana ${ }^{b}$, A.R. Jurelo ${ }^{c}$, M.S. Figueira ${ }^{d}$, G.G. Cabrera ${ }^{e}$, E.C. Siqueira ${ }^{\text {f,* }}$ \\ a Departamento de Física e Química, Universidade Estadual Paulista - UNESP, 15385-000, Ilha Solteira, SP, Brazil \\ b Departamento de Física, Universidad Técnica Federico Santa Maria, Av. Vicuña Mackenna, 3939, Santiago, Chile \\ c Departamento de Física, Universidade Estadual de Ponta Grossa - UEPG, 84030-000, Ponta Grossa, PR, Brazil \\ d Instituto de Física, Universidade Federal Fluminense, 24210-340, Niterói, RJ, Brazil \\ e Instituto de Física 'Gleb Wataghin', Universidade Estadual de Campinas - UNICAMP, 13083-859, Campinas, SP, Brazil \\ ${ }^{\mathrm{f}}$ Departamento de Física, Universidade Tecnológica Federal do Paraná - UTFPR, 84016-210, Ponta Grossa, PR, Brazil
}

\section{A R T I C L E I N F O}

\section{Article history:}

Received 28 August 2016

Received in revised form 26 November 2016

Accepted 29 November 2016

Available online 2 December 2016

Communicated by L. Ghivelder

\section{Keywords:}

Superconductivity

Ferromagnetism

Andreev transport

Aharonov-Bohm

Quantum dot

\begin{abstract}
A B S T R A C T
In this paper we study a double-path junction formed by a ferromagnetic and a superconductor lead. The first path connects the superconductor and ferromagnet directly while the second path connects these metals through a quantum dot. The whole system works as an Aharonov-Bohm interferometer allowing the study of the interference between these two paths under the presence of spin imbalance and Andreev bound states. We considered the effect of Fano interference on the electronic transmittance through the quantum dot and observed two regimes of conduction depending on the strength of the direct coupling. For the weak coupling regime, the transmittance presented the usual four resonances due to the Andreev bound states whereas for the strong coupling regime the profile was inverted and resonances became anti-resonances. However, even in the strong coupling regime it was possible to observe a central resonance due to the interference between the Andreev bound states. We have also studied the signatures of Fano interference on the average occupation within the quantum dot. The spin accumulation was analyzed and how it depends on the direct coupling and an external magnetic field applied to the system. The results obtained may be used in a possible experimental implementation of this system in order to probe spin related effects in ferromagnetic superconductor nanostructures.
\end{abstract}

(C) 2016 Elsevier B.V. All rights reserved.

\section{Introduction}

The relation between charge and spin degrees of freedom may be easily exploited in ferromagnetic/superconductor $(F / S)$ nanostructures [1-13] The ferromagnetism breaks the spin symmetry while superconductivity breaks the electron-hole symmetry. In a superconductor the Cooper pairs, responsible for the superconducting features, have the same energy separated from the excited states by an energy gap. When electrons from a ferromagnetic conductor are injected into a superconductor with energies smaller than the superconductor gap, the charge transference is still possible by means of Andreev reflection mechanism [2,14]. In this process, an electron of spin $\uparrow(\downarrow)$ and energy $E$ is reflected as a hole of spin $\downarrow(\uparrow)$ and energy $-E$; as a result, a Cooper pair is formed into the superconductor whose electrons are aligned in

\footnotetext{
* Corresponding author.

E-mail address: ecosta@utfpr.edu.br (E.C. Siqueira).
}

the singlet state. This mechanism allows one to control the flux of electrons by means of ferromagnet polarization and external potentials. This feature was used by Y. Zhu et al. [15] to write a spin in a quantum dot (QD) coupled to a ferromagnet and a superconductor. The authors showed that the spin within the QD may be changed by varying the bias and gate voltages even under the presence of electronic correlations.

Here we consider a generalization of the F-QD-S system by considering a second path for the electrons by assuming a direct coupling between $F$ and $S$. A schematic diagram of the system is illustrated in Fig. 1. The ferromagnetic lead is assumed to be biased by an electrical potential, $V$, while the superconductor is kept grounded. The coupling between the leads and the QD is assumed to be the same, $t_{d}$, and the direct coupling between $F$ and $S$ is determined by the parameter $t_{s f}$. The whole system presents a ring geometry working as an Aharonov-Bohm interferometer [16] (ABI). This kind of geometry has been extensively discussed in the literature, however, systems involving the presence of a superconductor lead have been given little attention. In this respect, 
Ref. [17] discusses the spin accumulation and spin polarized current for a normal metal and a superconductor coupled to the ABI. The spin accumulation is a result of a spin-orbit coupling assumed to occur within the QD. The Fano interference, expected to occur in ABIs, was addressed by Z. Yu et al. [18] also for normal metal and a superconductor coupled to the ring. The authors discussed the evolution of the transmittance from a resonance to an antiresonance due to the variation of the Fano factor. It is worth mentioning that in both works the electronic correlations within the QD were considered to be absent.

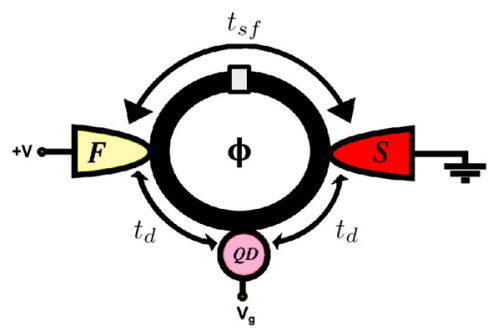

Fig. 1. Schematic diagram of the nanostructure considered in this report. The ferromagnetic lead $(F)$ and the superconductor lead $(S)$ are coupled by a quantum dot (QD) described by a single level $\varepsilon_{d}$ whose position can be changed by a gate voltage $V_{g}$. The ferromagnetic lead $(\mathrm{F})$ is also coupled directly to the superconductor lead $(S)$. Notice that the ferromagnetic lead is biased by an electric voltage $V$ while the superconductor is kept grounded. The couplings among all elements of the system are described by the intensities $t_{s f}$ and $t_{d}$. The whole system presents a ring geometry where the electrons of both paths interfere when hitting the superconductor. A magnetic flux $\phi$ piercing the ring is also considered and enters as a phase difference between the two paths taken by the electrons.
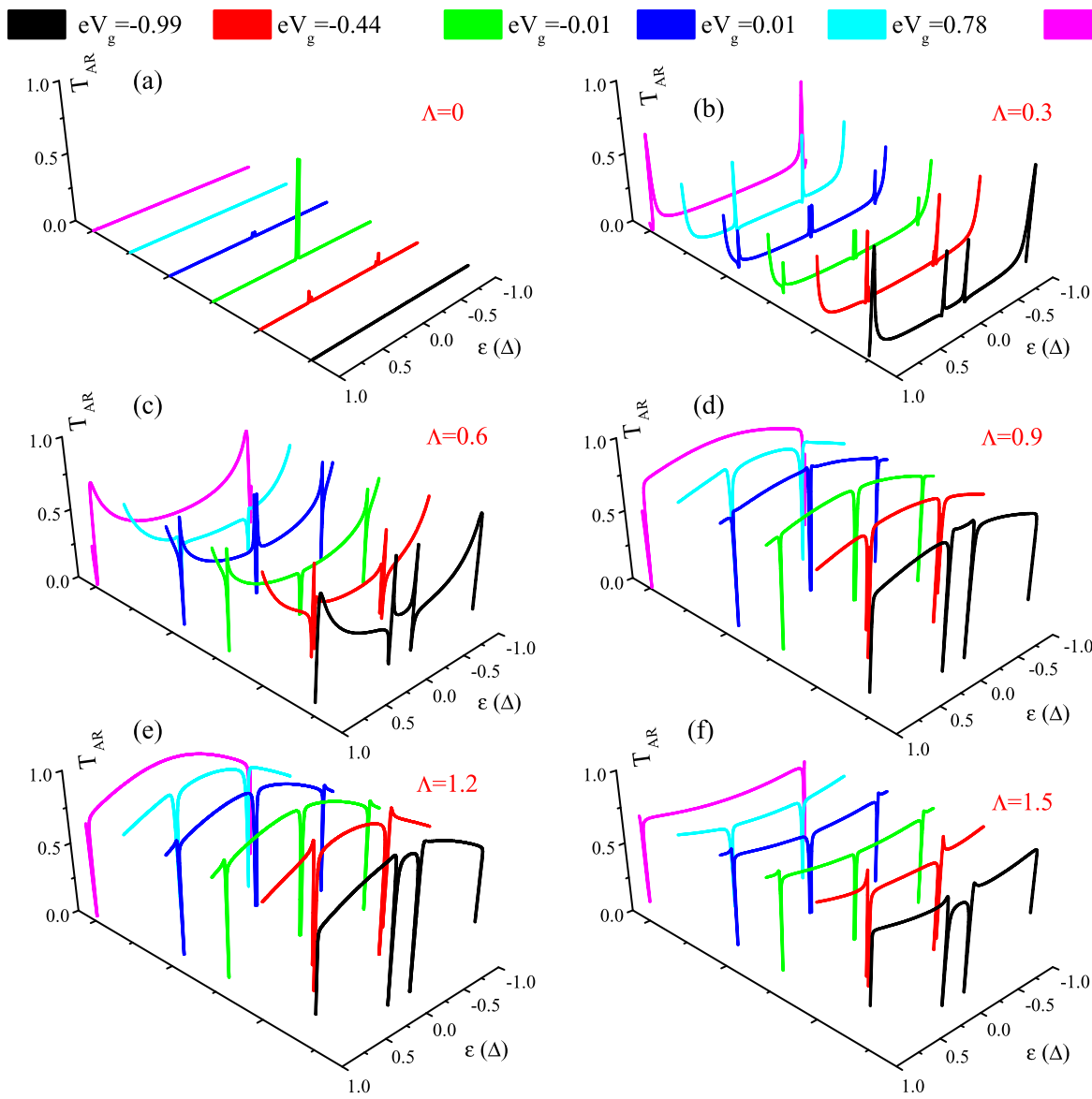

In contrast with these previous works, in this paper we consider the effect of the electronic correlations as well as the spin polarization provided by the ferromagnetic lead. The interplay among Andreev reflection, spin imbalance and the path interference, gives rise to a different pattern for the transmittance with a central resonance. In addition, we also verify if the spin writing effect, discussed in Ref. [15], is still present within the QD under the presence of the path interference and the magnetic flux through the ring.

This paper is organized as follows: in Sec. 2 we present the model for the system displayed in Fig. 2 and the physical quantities are determined by using the formalism of non-equilibrium Green's functions [19]. In Sec. 3 the results are presented and discussed. A summary and the main conclusions are presented in Sec. 4. An appendix is included with the mathematical details of the calculations.

\section{Model and formulation}

The system illustrated in Fig. 1 is described by the following Hamiltonian:

$$
\begin{aligned}
\hat{\mathcal{H}}= & \sum_{\mathbf{k} \sigma}\left(\varepsilon_{\mathbf{k}}-\operatorname{sgn}(\sigma) h-\mu_{f}\right) \hat{c}_{\mathbf{k} \sigma}^{\dagger} \hat{c}_{\mathbf{k} \sigma} \\
& +\sum_{\mathbf{k} \sigma}\left(\varepsilon_{\mathbf{k}}-\mu_{S}\right) \hat{c}_{\mathbf{k} \sigma}^{\dagger} \hat{c}_{\mathbf{k} \sigma}+\sum_{\mathbf{k}}\left[\Delta_{s}^{*} \hat{c}_{\mathbf{k} \uparrow}^{\dagger} \hat{c}_{-\mathbf{k} \downarrow}^{\dagger}+\text { H.c. }\right] \\
& +\sum_{\sigma}\left(\varepsilon_{d}-e V_{g}\right) \hat{d}_{\sigma}^{\dagger} \hat{d}_{\sigma}+U \hat{n}_{d \uparrow} \hat{n}_{d \downarrow}
\end{aligned}
$$




$$
\begin{aligned}
& +\sum_{\mathbf{k} \sigma}\left[t_{d} \hat{c}_{\mathbf{k} \sigma}^{\dagger} \hat{d}_{\sigma}+\text { H.c. }\right]+\sum_{\mathbf{k} \sigma}\left[t_{d} e^{i \phi} \hat{d}_{\sigma}^{\dagger} \hat{c}_{\mathbf{k} \sigma}+\text { H.c. }\right] \\
& +\sum_{\mathbf{k} \mathbf{q} \sigma}\left[t_{f s} \hat{c}_{\mathbf{k} \sigma}^{\dagger} \hat{c}_{\mathbf{q} \sigma}+\text { H.c. }\right] .
\end{aligned}
$$

The ferromagnetic lead is modeled by a Stoner mean-field model [20] in the first line of Eq. (1). The spin up (down) band is shifted by the mean-field $+h(-h)$ and the chemical potential $\mu_{F}$ is fixed by the bias $V$, applied in $F$. Notice that it is used a free electron dispersion $\varepsilon_{\mathbf{k}}=\hbar^{2} k^{2} / 2 m$ is used. The superconductor is modeled by a BCS model [21] with $\Delta_{S}$ being the mean-field pair potential, assumed as a constant for simplicity. The QD is described by a Hubbard dot [see Eqs. (A.4) to (A.9) from Appendix A] with $U$ being the intensity of the electronic correlation. The spin degenerated level $\varepsilon_{d}$ is aligned to the superconductor chemical potential $\left(\mu_{s}\right)$ which is set to zero as the ground. The position of $\varepsilon_{d}$ may be controlled by means of a gate voltage, $V_{g}$, applied directly to the QD. The first sum in the fourth line describes the tunneling process between the ferromagnetic lead and the QD while the second sum describes the tunneling between the QD and the superconductor lead. The phase $\phi$ appearing in this term takes into account the presence of a magnetic flux through the ring formed by the two paths taken by the electrons from the ferromagnetic lead to the superconductor lead. The last term describes the direct tunneling between the leads.

By using non-equilibrium Green's function method [19] it is possible to cast all the physical quantities in terms of the retarded/advanced Green's functions, $\mathbf{G}^{r / a}$, and the "lesser" Green's function, $\mathbf{G}^{<}$. These Green's functions present the following structure,

$$
\mathbf{G}^{r / a,<}(\varepsilon)=\left[\begin{array}{lll}
\mathbf{G}_{f f}^{r / a,<}{ }_{(\varepsilon)} & \mathbf{G}_{f d}^{r / a,<}{ }_{(\varepsilon)} & \mathbf{G}_{f s}^{r / a,<}{ }_{(\varepsilon)} \\
\mathbf{G}_{d f}^{r / a,<}{ }_{(\varepsilon)} & \mathbf{G}_{d d}^{r / a,<}{ }_{(\varepsilon)} & \mathbf{G}_{d s}^{r / a,<}{ }_{(\varepsilon)} \\
\mathbf{G}_{s f}^{r / a,<}{ }_{(\varepsilon)} & \mathbf{G}_{s d}^{r / a,<}{ }_{(\varepsilon)} & \mathbf{G}_{s s}^{r / a,<}{ }_{(\varepsilon)}
\end{array}\right]
$$

where the indexes $f, d$ and $s$ correspond to the ferromagnetic lead, quantum dot and superconductor lead subspaces. Each matrix element of Eq. (2) is itself a $4 \times 4$ matrix in the Nambu space [22,23]. In fact, in order to take both ferromagnetism and superconductivity on an equal footing, it is necessary to work within a space composed by a tensor product between spin and electron-hole states.

The electrical current may be expressed as follows:

$I=\frac{4 e}{h} \int T_{A R}(\varepsilon)\left(f_{f}-\bar{f}_{f}\right) d \varepsilon$,

where $f_{f}=f(\varepsilon-e V)$ and $\bar{f}_{f}=f(\varepsilon+e V)$ are the electron and hole Fermi functions, respectively, for the ferromagnetic lead. These Fermi functions depend on the bias potential, $e V$, applied to the ferromagnet electrode. The expression for $T_{A R}(\varepsilon)$ is given in Appendix A.

The average occupation number (in the QD) is determined by the expression:

$n_{d \uparrow(\downarrow)}=\int\left[\mathbf{G}_{d d}^{<}\left(\varepsilon, n_{d \uparrow}, n_{d \downarrow}\right)\right]_{11(33)} d \varepsilon$,

where $\mathbf{G}_{d d}^{<}$is the "lesser" QD Green's function. Notice that the indexes 11(33) means taking the matrix element 11(33) of the $4 \times 4$ matrix corresponding to the spin up (down) electron. This Green's function may be calculated by taking the QD Green's function from the general Keldysh equation $\mathbf{G}^{<}=\mathbf{G}^{r} \mathbf{g}^{r-1} \mathbf{g}^{<} \mathbf{g}^{a-1} \mathbf{G}^{a}$, with $\mathbf{g}^{<}$being the "lesser" Green's function for the system when the QD is decoupled from the leads. In Appendix A, the expressions for $\mathbf{g}^{<}$ and $\mathbf{g}^{r / a}$ are shown. It is worth noting that $\mathbf{G}_{d d}^{<}$is dependent on $n_{d \uparrow}$ and $n_{d \downarrow}$ which means that the Eq. (4) must be solved in a self-consistent way.
The Andreev transmittance and the occupation number are studied in terms of the coupling strengths between the QD and leads, $\Gamma_{\eta}=2 \pi \mathcal{D}_{\eta}\left(\varepsilon_{F}\right)\left|t_{\eta}\right|^{2}$, with $\eta=s$, $f$. It is assumed that the normal density of states, $\mathcal{D}_{\eta}\left(\varepsilon_{F}\right)$, solved at the Fermi level, is the same for the ferromagnet and superconductor leads. The direct coupling between ferromagnetic and superconductor leads is modeled by the dimensionless parameter, $\Lambda=\pi\left|t_{s f}\right| \mathcal{D}_{\eta}\left(\varepsilon_{F}\right)$. In addition to the coupling parameters, we also define the transport polarization, $P$, as follows:

$P=\frac{\Gamma_{f \uparrow}-\Gamma_{f \downarrow}}{\Gamma_{f \uparrow}+\Gamma_{f \downarrow}}$,

where $\Gamma_{f \uparrow(\downarrow)}=\Gamma_{f}(1 \pm \sigma P)$ is the coupling strength per spin such that $\Gamma_{f}=\left(\Gamma_{f \uparrow}+\Gamma_{f \downarrow}\right) / 2$.

By using above definitions, we study the transmittance and average occupation number with $\Gamma_{\eta}, \Lambda$ and $P$ taken as independent parameters.

\section{Results and discussion}

In this section we present the results for transmittance and spin occupation within the quantum dot. All the parameters are scaled by the superconductor gap which is the natural energy scale for this system.

\subsection{Fano signatures in the transmittance curves}

The nanodevice illustrated in Fig. 2 is constituted by a quantum dot (QD) and a direct coupling between the leads. This coupling is characterized by the dimensionless parameter, $\Lambda$. In this section, the role of the direct coupling in the transmittance through the nanodevice is addressed. The direct coupling gives rise to the interference between the two paths taken by the electrons either by a direct tunneling between the leads or through the QD. This kind of interference is well-known as the Fano effect and has been extensively studied in previous works $[16,24]$. In the present context, the superconductor introduces an additional interference channel between electron and hole states, which gives rise to a different Fano profile as compared to the one appearing in nanostructures made with normal metals. These states appear as two resonances located in symmetric positions at $\pm \varepsilon$. In Fig. 2 some transmittance curves are shown for different values of the lead-lead coupling, $\Lambda$, and the gate voltage, $V_{g}$, applied to the QD.

In Fig. 2(a) displays the transmittance for $\Lambda=0$. The transmittance achieves its maximum value for $e V_{g}=0$, where the QD level is aligned to the superconductor chemical potential, being strongly suppressed as $e V_{g}$ is increased to values close to the superconductor gap. For $\Lambda=0.3$, the Andreev transport is improved and the resonances may be observed for all values of $\varepsilon$, this is illustrated in Fig. 2(b). For $e V_{g}>0$, two additional peaks appear in the curves due to the intradot electronic correlations. These resonances are located for $\varepsilon= \pm\left(e V_{g}+U\right)$ where $U$ is the strength of the intradot interaction. The resonances appear as a result of the constructive interference between the electron and hole states for different spins due to the finite spin polarization $(P=0.5)$. When $\Lambda$ is increased from zero, an additional interference channel appears due to the different paths taken by the electron and holes and therefore the conditions of maximum of the transmittance change. This is observed for $\Lambda=0.6$ in which the transmittance curves exhibit an intermediate profile for all values of $e V_{g}$, as shown in Fig. 2(c). We can see that there is a mixture of resonance and antiresonance due to the Fano interference. As the lead-lead coupling is further increased, the resonances are replaced by anti-resonances at the same values of $\varepsilon$, as is illustrated in Figs. 2(d), 2(e) and 2(f).

The physics occurring in the superconductor gap scale is ruled by the Fano interference as $\Lambda$ increases. However, within the energy scale fixed by $\Gamma_{s}$, the interference between electron-hole 

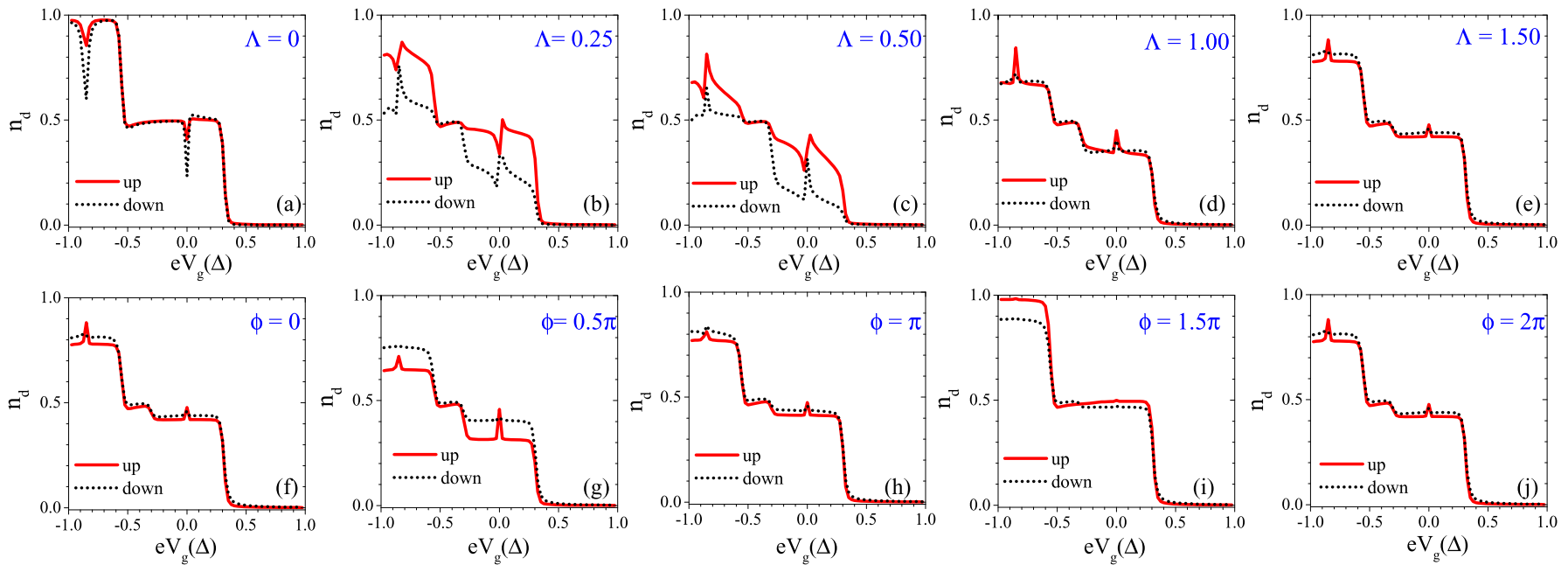

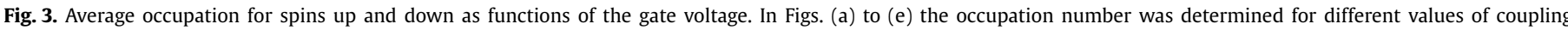

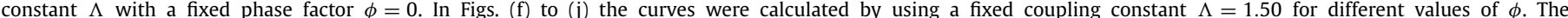

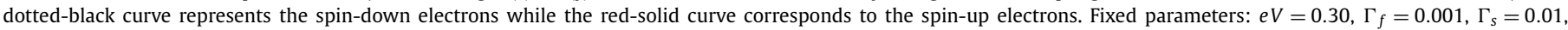
$P=0.5, \mathcal{U}=0.86$ and $k_{B} T=0.1$. All parameters are given in gap superconductor units.

states introduces an additional pattern into the $T_{A R}$ curves. This may be observed by comparing Figs. 2(g), 2(h) and 2(i), where the transmittance for $e V_{g}=-0.35$ is shown for the range $-0.05<\varepsilon<$ +0.05 . For $\Lambda=0$, the Andreev bound states appear as two equidistant resonances, black curve in Fig. $2(\mathrm{~g})$. As $\Lambda$ is set to 0.3 , these resonances are smoothed but the Andreev pattern is still evident. For $\Lambda>0.3$, the path interference becomes dominant and the two peaks are converted into two anti-resonances at the same values of $\varepsilon$. This effect is expected from the Fano interference but, in this case, a non-expected central resonance appears at $\varepsilon=0$. This behavior remains for all values of $\Lambda$ as shown in Figs. 2(h) and 2(i). This new central resonance is a result of the combination of the Fano effect and the Andreev bound states. It is worth noting that this resonance is preserved even under the presence of electronic correlations and spin polarization of the ferromagnetic lead.

\subsection{Fano signatures in the occupation number}

The electron's spin in a QD coupled to ferromagnetic and superconductor leads can be controlled by means of a gate voltage. In fact, the ferromagnet injects spin up and spin down electrons at different rates into the QD while the superconductor collects such electrons at the same rate. As a result, there is a spin accumulation within the QD which is dependent on the gate and bias voltages. Such effect may be interesting in devices exploring spintronic properties. In order to implement such nanostructure in experiments, one may use a Scanning Tunneling Microscopy (STM) in which an adatom is adsorbed in a superconductor metal host while a ferromagnetic STM tip may be used to inject the electrons into the host. In a realistic experiment, the STM tip may be coupled directly to the superconductor and the geometry of the system reduced to the one studied in this work. Thus, in this section we consider the occupation number within the QD as a function of the gate voltage, $V_{g}$, and the coupling parameter, $\Lambda$.

In Fig. 3, the average occupation, $n_{d \sigma}$, for spins up and down, are shown for a fixed bias voltage, $e V=+0.30$, applied to the ferromagnetic lead. Since the ferromagnet polarization was set to $P=0.5$, spin up electrons were injected into the $\mathrm{QD}$ at higher rates than spin down electrons. Notice that both spin up and spin down curves present a three step feature due to the electronic correlations within the QD. In fact, the electronic correlations raise the spin degeneracy and the bare QD levels are split into two levels located at $\varepsilon_{d}$ and $\varepsilon_{d}+U$. The position of the bare QD level is determined by the value of the gate voltage applied to the QD. As the gate voltage is reduced from its maximum value $\left(e V_{g}=+\Delta\right)$, the first level is pulled down until it is to be aligned to the ferromagnet chemical potential, set by the bias voltage to $\mu_{f}=+0.30$, where the first step appears in the occupation number. In this case, the occupation number is equal to unity on average. As the gate voltage is further reduced, a second step appears for $e V_{g}=-\left(\mu_{f}-U\right) \approx-0.56$. At this point, the second level aligns to the ferromagnet chemical potential and the occupation number jumps to 2 electrons on average. Superimposed on this pattern, there are two Andreev resonances located to $e V_{g}=0$ and $e V_{g}=-0.86$, which produce a fluctuation in the occupation number. This behavior manifests either as anti-resonances in Fig. 3(a)-(b) or resonances in Fig. 3(d)-(e).

The dependence of the occupation number on $\Lambda$ is illustrated in Figs. 3(a) to 3(e). For $\Lambda=0$ the difference between $n_{d \uparrow}$ and $n_{d \downarrow}$ is maximum at the antiresonances located in $e V_{g}=0$ and $e V_{g}=-0.86$. For $\Lambda=0.25$ and $\Lambda=0.50$, illustrated by Figs. 3(b) and 3(c), respectively, the Fano interference appears in the occupation curves being stronger for $n_{d \downarrow}$ curves (dotted black curves). These curves exhibit an intermediate behavior between anti-resonance and resonance and the difference between the occupation number occurs for some intermediate values of $e V_{g}$. At the same points, at which Fig. 3(a) show a well localized antiresonance, in Figs. 3(b) and 3(c) the occupations present the same value. For $\Lambda>1$, as shown in Figs. 3(d) and 3(e), the pattern is inverted and the anti-resonances are completely converted into resonances at the same points as Fig. 3(a). However, the spin up occupation still remains higher than the spin down occupation.

We also considered the possibility of a transversal magnetic flux through the ring formed by the two paths taken by the electrons. The magnetic flux enters as a phase difference $\phi$, taken as the independent parameter. The average occupations were determined for some values of $\phi$, as illustrated in Figs. 3(f) to 3(j). It has been used the same set of parameters as in Fig. 3(e). For $\phi=\pi / 2$, the spin accumulation is inverted in the second and fourth plateau, as shown in Fig. 3(g). As $\phi$ is further increased, the spin accumulation starts to be reduced and, for $\phi=\pi$, the occupation curves coincide for most values of $e V_{g}$, as shown in Fig. 3(h). The Andreev reflection resonances are also dependent on $\phi$ being almost suppressed for $\phi=3 \pi / 2$ as illustrated in Fig. 3(i). Finally, for $\phi=2 \pi$, the same pattern as $\phi=0$ is recovered again as may be observed in Fig. $3(\mathrm{j})$. These results show that the spin accumulation within 
the QD is strongly dependent on the magnetic flux and, in order to implement such a device, it is necessary either to eliminate any magnetic field effects from the system or to have the control of the magnetic flux.

\section{Conclusions}

The Fano interference and spin accumulation in the system illustrated in Fig. 1 were addressed in this work. The Fano effect was shown to present a different pattern in comparison to conventional systems due to the interplay with the Andreev bound states. In fact, it was possible to observe a central resonance at $\varepsilon=0$ in the transmittance curves even for a strong coupling between the leads and under the presence of electronic correlations within the QD. The spin accumulation at the QD was also studied since the F-QD-S system allows one to write a spin by using a combined set of electrical potentials. Such an effect is interesting for spintronic applications. Here we have analyzed the influence of a direct coupling and a transversal magnetic flux through the system on the QD average spin. The results show that the direct coupling and the magnetic flux can modify the spin accumulation within the QD. For moderate values of $\Lambda$, as shown in Figs. 3(b) and 3(c), the presence of the direct coupling can favor the spin accumulation since the spin up and spin down populations are different in a wider range of gate voltages. The magnetic flux can also be used to help in the spin accumulation by varying its flux in a proper value.

The condition for subgap current $(\Delta>U)$ is not common to be found in experimental setups involving a QD coupled to a superconductor. However, as pointed out by I. Weymann \& P. Trocha in Ref. [25], there are some superconductors [26,27] with energy gap as large as milli-electron volts while the charging energy may be reduced to values smaller than the superconductor gap. In this way, the experimental verification of the results presented in this work may be done with the current technology.

\section{Acknowledgements}

P. A. O. acknowledges support from FONDECYT grant No. 1140571 and CONICYT ACT 1204.

\section{Appendix A. Green's function expressions}

The transmittance, $T_{A R}$, appearing in Eq. (3) is given by,

$T_{A R}=\operatorname{Re}\left\{\operatorname{Tr}\left[\mathbf{Q}_{e} \boldsymbol{\Sigma} \mathbf{G}^{r} \mathbf{Q}_{h}\left(\mathbf{g}^{r-1}-\mathbf{g}^{a-1}\right) \mathbf{G}^{a}\right]\right\}$,

where "Re" stands for the real part of the complex expression between $\{\cdots\}$ while "Tr" stands for the trace of the matricial product. The matrices $\mathbf{Q}_{e}$ and $\mathbf{Q}_{h}$ are the charge matrices for electrons and holes, respectively. These are written as follows:

$\mathbf{Q}_{e, h}=\left[\begin{array}{ccc}\mathbf{q}_{e, h} & \mathbf{0} & \mathbf{0} \\ \mathbf{0} & \mathbf{0} & \mathbf{0} \\ \mathbf{0} & \mathbf{0} & \mathbf{0}\end{array}\right]$

where each matrix element in Eq. (A.2) is itself a $4 \times 4$ matrix in Nambu space. This is a result of the tensor product of electron-hole and spin spaces. In fact, the matrices $\mathbf{q}_{e}$ and $\mathbf{q}_{h}$ are written as $\mathbf{q}_{e, h}= \pm(1 / 2) \tau_{0} \otimes\left(\tau_{0} \pm \tau_{3}\right)$ where $\tau_{i}$, with $i=1,2,3$, are the Pauli matrices and $\tau_{0}$ is the identity matrix. The coupling matrix $\boldsymbol{\Sigma}$ is written as

$\boldsymbol{\Sigma}=\left[\begin{array}{ccc}\mathbf{0} & \mathbf{T}_{f d} & \mathbf{T}_{f s} \\ \mathbf{T}_{f d}^{\dagger} & \mathbf{0} & \mathbf{T}_{d s}^{\dagger} \\ \mathbf{T}_{f s}^{\dagger} & \mathbf{T}_{s d} & \mathbf{0}\end{array}\right]$

where each matrix element is a $4 \times 4$ matrix, $\mathbf{T}_{\alpha \beta}=t_{\alpha \beta} \mathbf{q}_{e}+t_{\alpha \beta}^{*} \mathbf{q}_{h}$ with $\alpha, \beta=s, f, d$.
The retarded/advanced Green's functions appearing in the transmittance equation are determined by Dyson's equation

$\mathbf{G}^{r / a}=\left\{\mathbf{g}^{(r / a)-1}-\mathbf{\Sigma}\right\}^{-1}$

with $\boldsymbol{\Sigma}$ being given by Eq. (A.3).

The Green's functions $\mathbf{g}^{(r / a)}$ have the following form,

$\mathbf{g}^{(r / a)}=\left[\begin{array}{ccc}\mathbf{g}_{f f}^{(r / a)} & \mathbf{0} & \mathbf{0} \\ \mathbf{0} & \mathbf{g}_{d d}^{(r / a)} & \mathbf{0} \\ \mathbf{0} & \mathbf{0} & \mathbf{g}_{s s}^{(r / a)}\end{array}\right]$,

where each matrix element of Eq. (A.5) is given by $\mathbf{g}_{f f}^{(r / a)}=$ $\mp\left(i \Lambda / t_{f s}\right) \mathbf{P}$, with $\mathbf{P}$ being the polarization matrix, $\mathbf{P}=(1+P) \mathbf{q}_{e}-$ $(1-P) \mathbf{q}_{h} ; \mathbf{g}_{s s}^{(r / a)}=\mp\left(i \Lambda_{s} / t_{f s}\right) \boldsymbol{\Delta}$ where the matrix $\boldsymbol{\Delta}$ is defined as $\Delta=\varrho(\varepsilon)\left[\begin{array}{cccc}1 & \Delta / \varepsilon & 0 & 0 \\ \Delta / \varepsilon & 1 & 0 & 0 \\ 0 & 0 & 1 & \Delta / \varepsilon \\ 0 & 0 & \Delta / \varepsilon & 1\end{array}\right]$,

with $\varrho(\varepsilon)=\frac{|\varepsilon| \vartheta(\varepsilon-|\Delta|)}{\sqrt{\varepsilon^{2}-\Delta^{2}}}-\frac{i \varepsilon \vartheta(\Delta-|\varepsilon|)}{\sqrt{\Delta^{2}-\varepsilon^{2}}}$ being the modified density of states with the imaginary term - for $\varepsilon<\Delta-$ accounting for the Andreev bound states.

The Green's function $\mathbf{g}_{d d}^{(r / a)}$ corresponds to the QD contribution under the presence of Coulomb correlations. We have used the Hubbard-I approximation to treat the electronic correlations within the QD. This approximation is valid in the weak-coupling regime $[28,29]$ which is obtained the limit $U / \Gamma \gg 1$. In fact, the values $U=0.85$ and $\Gamma=0.001$ lead to a ratio $U / \Gamma \sim 850$, which is a safe limit for the validity of this approximation. The ferromagnet polarization reduces the spin fluctuations within the QD which also helps the applicability of this approximation [30-32].

Explicitly, $\mathbf{g}_{d d}$ reads

$\mathbf{g}_{d d}^{(r / a)}=\left[\begin{array}{cccc}g_{e e, \uparrow \uparrow}^{r / a} & 0 & 0 & 0 \\ 0 & g_{h h, \downarrow \downarrow}^{r / a} & 0 & 0 \\ 0 & 0 & g_{e e, \downarrow \downarrow}^{r / a} & 0 \\ 0 & 0 & 0 & g_{h h, \uparrow \uparrow}^{r / a}\end{array}\right]$,

where $g_{e e, \sigma \sigma}^{r / a}$ and $g_{h h, \sigma \sigma}^{r / a}$ are the electron and hole matrix elements, respectively, whose expression is given by:

$g_{e e, \sigma \sigma}^{r / a}=\frac{1-\left\langle\hat{n}_{\bar{\sigma}}\right\rangle}{\varepsilon-\zeta \pm i 0}+\frac{\left\langle\hat{n}_{\bar{\sigma}}\right\rangle}{\varepsilon-(\zeta+U) \pm i 0}$,

and

$g_{h h, \sigma \sigma}^{r / a}=\frac{1-\left\langle\hat{n}_{\bar{\sigma}}\right\rangle}{\varepsilon+\zeta \pm i 0}+\frac{\left\langle\hat{n}_{\bar{\sigma}}\right\rangle}{\varepsilon+(\zeta+U) \pm i 0}$,

in which $\sigma=\uparrow, \downarrow$ stands for the spin component and $\bar{\sigma}$ stands for the opposite of $\sigma ; \zeta=\varepsilon_{d}-e V_{g}$ with $\varepsilon_{d}$ being the bare QD level and $V_{g}$ is the gate voltage. Notice that Eqs. (A.7) and (A.8) are dependent on the average occupation $\hat{n}_{\sigma}$ on the QD.

Finally, the lesser $\mathbf{g}^{<}$Green's function is given by the expression

$\mathbf{g}^{<}=\left[\begin{array}{ccc}\mathbf{g}_{f f}^{<} & \mathbf{0} & \mathbf{0} \\ \mathbf{0} & \mathbf{0} & \mathbf{0} \\ \mathbf{0} & \mathbf{0} & \mathbf{g}_{s s}^{<}\end{array}\right]$

with $\mathbf{g}_{\alpha \alpha}^{<}=\mathbf{F}_{\alpha}\left(\mathbf{g}_{\alpha \alpha}^{a}-\mathbf{g}_{\alpha \alpha}^{r}\right)$ with $\mathbf{F}_{\alpha}=f_{\alpha} \mathbf{q}_{e}-\bar{f}_{\alpha} \mathbf{q}_{h}$ is the Fermi matrix with $f_{\alpha}=f\left(\varepsilon-e V_{\alpha}\right)$ and $\bar{f}_{\alpha}=f\left(\varepsilon+e V_{\alpha}\right)$ being the electron and hole Fermi distributions for the lead $\alpha=f, s$. 


\section{References}

[1] A.I. Buzdin, Proximity effects in superconductor-ferromagnet heterostructures, Rev. Mod. Phys. 77 (2005) 935-976.

[2] I. Žutić, J. Fabian, S. Das Sarma, Spintronics: fundamentals and applications, Rev. Mod. Phys. 76 (2004) 323-410.

[3] I. Weymann, K.P. Wójcik, Andreev transport in a correlated ferromagnetquantum-dot-superconductor device, Phys. Rev. B 92 (2015) 245307.

4] L. Li, Z. Cao, H.-G. Luo, F.-C. Zhang, W.-Q. Chen, Fano resonance in a norma metal/ferromagnet-quantum dot-superconductor device, Phys. Rev. B 92 (2015) 195155.

[5] E.C. Siqueira, G.G. Cabrera, Magnetoresistance and transistor-like behavior of a double quantum-dot via crossed Andreev reflections, J. Appl. Phys. 111 (2012) 113905.

[6] E.C. Siqueira, P.A. Orellana, R.C. Cestari, M.S. Figueira, G.G. Cabrera, Fano effect and Andreev bound states in a hybrid superconductor ferromagnetic nanostructure, Phys. Lett. A 379 (2015) 2524-2529.

[7] P. Trocha, J. Barnaś, Spin-polarized Andreev transport influenced by Coulomb repulsion through a two-quantum-dot system, Phys. Rev. B 89 (2014) 245418.

[8] K. Bocian, W. Rudziński, Influence of intradot Coulomb correlations on the Andreev reflection in a ferromagnetic metal/quantum dot/superconductor hybrid junction, Eur. Phys. J. B 86 (2013) 1-7.

[9] G.-Y. Yi, X.-Q. Wang, H.-N. Wu, W.-J. Gong, Nonlocal magnetic configuration controlling realized in a triple-quantum-dot Josephson junction, Physica E, Low-Dimens. Syst. Nanostruct. 81 (2016) 26-30.

[10] K.P. Wójcik, I. Weymann, Proximity effect on spin-dependent conductance and thermopower of correlated quantum dots, Phys. Rev. B 89 (2014) 165303.

[11] R. Žitko, J.S. Lim, R. López, R. Aguado, Shiba states and zero-bias anomalies in the hybrid normal-superconductor Anderson model, Phys. Rev. B 91 (2015) 045441.

[12] J. Bauer, A. Oguri, A.C. Hewson, Spectral properties of locally correlated electrons in a Bardeen-Cooper-Schrieffer superconductor, J. Phys. Condens. Matter 19 (2007) 486211.

[13] T. Domański, I. Weymann, M. Barańska, G. Górski, Constructive influence of the induced electron pairing on the Kondo state, Nature 6 (2016) 23336.

[14] A.F. Andreev, The thermal conductivity of the intermediate state in superconductors, Sov. Phys. JETP 19 (1964) 1228-1231.

[15] Y. Zhu, T.-h. Lin, Q.-f. Sun, Writing spin in a quantum dot with ferromagnetic and superconducting electrodes, Phys. Rev. B 69 (2004) 121302.

[16] A.E. Miroshnichenko, S. Flach, Y.S. Kivshar, Fano resonances in nanoscale structures, Rev. Mod. Phys. 82 (2010) 2257-2298.
17] H. Pan, Y. Cui, H, Wang, R. Wang, Spin-polarized Andreev reflection and spin accumulation in a quantum-dot Aharonov-Bohm interferometer with spinorbit interaction effects, J. Appl. Phys. 110 (2011) 033706.

[18] Z. Yu, M. Zhong-Shui, S. Qing-Feng, L. Tsung-Han, Andreev-Fano effect in hybrid normal-metal/superconductor interferometer, Commun. Theor. Phys. 42 (2004) 155.

[19] L.V. Keldysh, Diagram technique for nonequilibrium processes, Sov. Phys. JETP 20 (1965) 1018-1026.

[20] P. Fazekas, Lecture Notes on Electron Correlations and Magnetism, World Scientific, Singapore, 1999.

[21] J. Bardeen, L.N. Cooper, J.R. Schrieffer, Microscopic theory of superconductivity, Phys. Rev. 106 (1957) 162-164.

[22] Z.Y. Zeng, B. Li, F. Claro, Electronic transport in hybrid mesoscopic structures: a nonequilibrium green function approach, Phys. Rev. B 68 (2003) 115319.

[23] Z. Zeng, B. Li, F. Claro, Electron transport in interacting hybrid mesoscopic systems, Eur. Phys. J. B 32 (2003) 401-405.

[24] U. Fano, Sullo spettro di assorbimento dei gas nobili presso il limite dello spettro da arco, Nuovo Cimento 12 (1935) 154-161.

[25] I. Weymann, P. Trocha, Superconducting proximity effect and zero-bias anomaly in transport through quantum dots weakly attached to ferromagnetic leads, Phys. Rev. B 89 (2014) 115305.

[26] J. Nagamatsu, N. Nakagawa, T. Muranaka, Y. Zenitani, J. Akimitsu, Superconductivity at $39 \mathrm{~K}$ in magnesium diboride, Nature 410 (2001) 63-64.

[27] B.W. Heinrich, L. Braun, J.I. Pascual, K.J. Franke, Protection of excited spin states by a superconducting energy gap, Nat. Phys. 9 (2013) 765-768.

[28] J.C. Cuevas, A. Levy Yeyati, A. Martín-Rodero, Kondo effect in normalsuperconductor quantum dots, Phys. Rev. B 63 (2001) 094515.

[29] S. de Franceschi, L. Kouwenhoven, C. Schönenberger, W. Werndorfer, Hybrid superconductor-quantum dot devices, Nat. Nanotechnol. 5 (2010).

[30] J. Martinek, Y. Utsumi, H. Imamura, J. Barnaś, S. Maekawa, J. König, G. Schön, Kondo effect in quantum dots coupled to ferromagnetic leads, Phys. Rev. Lett. 91 (2003) 127203.

[31] J. Martinek, M. Sindel, L. Borda, J. Barnaś, J. König, G. Schön, J. von Delft, Kondo effect in the presence of itinerant-electron ferromagnetism studied with the numerical renormalization group method, Phys. Rev. Lett. 91 (2003) 247202.

[32] S. Andergassen, V. Meden, H. Schoeller, J. Splettstoesser, M.R. Wegewijs, Charge transport through single molecules, quantum dots and quantum wires, Nanotechnology 21 (2010) 272001. 